Bond University

Research Repository

\title{
Benefits and barriers of cancer practitioners discussing physical activity with their cancer patients
}

Keogh, Justin W L; Olsen, Alicia; Climstein, Michael; Sargeant, Sally; Jones, Lynnette

Published in:

Journal of Cancer Education

DOI:

10.1007/s13187-015-0893-1

Licence:

Unspecified

Link to output in Bond University research repository.

Recommended citation(APA):

Keogh, J. W. L., Olsen, A., Climstein, M., Sargeant, S., \& Jones, L. (2017). Benefits and barriers of cancer practitioners discussing physical activity with their cancer patients. Journal of Cancer Education, 32(1), 11-15. https://doi.org/10.1007/s13187-015-0893-1

\section{General rights}

Copyright and moral rights for the publications made accessible in the public portal are retained by the authors and/or other copyright owners and it is a condition of accessing publications that users recognise and abide by the legal requirements associated with these rights.

For more information, or if you believe that this document breaches copyright, please contact the Bond University research repository coordinator. 


\title{
Benefits and barriers of cancer practitioners discussing physical activity with their cancer patients
}

\begin{abstract}
Our aim was to synthesise the existing empirical literature and theoretical perspectives on the physical activity promotion practices and determinants of cancer clinicians and health professionals. We conducted a narrative review of theory and evidence to develop practice recommendations for improving the promotion of physical activity to cancer patients. Surveyed health professionals were aware of many benefits of physical activity for their cancer patients, although only $\sim 40 \%$ promoted physical activity to selected cancer patients. Walking was most commonly promoted form of PA, with this promoted to assist patients control their weight and cardiovascular health risk. Barriers to promotion of physical activity included lack of time and knowledge of PA and behaviour change skills. Health professionals appear interested in promoting PA to their cancer patients, yet encounter several barriers. Further research is warranted to assist health professionals improve their PA promotion. An adapted reflective-impulsive model of social behaviour shows promise for assisting health professionals overcome barriers and provides an evidence-based theoretical framework for improving communication with patients. Universities, hospitals and/or healthcare accreditation organisations also have important roles to play in assisting health professionals improve their promotion of physical activity to patients.
\end{abstract}

Keywords: cancer; counselling; exercise; oncology; physical activity. 


\section{Introduction}

There is growing evidence demonstrating that physical activity (PA), be it aerobic, resistance (strength) or a combination thereof produces physical and psychosocial benefits $[7,11,16]$ that counteract many of the adverse effects of common cancer treatments $[8,17]$. Such research has led professional organisations, including the American Cancer Society, to develop position statements outlining the importance of PA and recommending that physically inactive patients become more active. Specifically, guidelines recommend that cancer patients perform 150 minutes of aerobic and 60 minutes of resistance training per week [16].

Health professions (e.g., general practitioners, oncologists and oncology nurses) are expected to provide high-quality, evidence-based education and counselling to their patients in relation to the potential risks and benefits of cancer screening and/or treatment [20]. However, studies have shown that health professionals report numerous barriers preventing them from discussing PA with their patients, and most importantly assisting them to make the necessary behavioural changes to accommodate recommended PA levels [5, 6, 18, 21]. We offer a narrative review of the relevant empirical work and propose practice recommendations for health professions, hospitals and universities for improving the promotion of PA to cancer patients.

\section{Discussing physical activity with cancer patients}

A recent study revealed that most cancer patients do not adhere to the PA guidelines of the American Cancer Society, and many are less active than their age-matched, non-cancer peers [10]. In light of research demonstrating the benefits of PA for cancer patients, it is clear that health professionals have a duty of care to more regularly discuss PA during their consultations. There is now considerable research that has examined the determinants of cancer patients’ PA levels in an attempt to investigate possible ways to increase their PA [9, 19, 13, 14]. Surprisingly, very little of this research has focused on the role of the health professional in supporting the PA behaviour of the patients $[18,15]$. A summary of the main issues facing cancer patients post-diagnosis, the benefits of regular PA and the possible role of health professionals in assisting their patients becoming more physically active is summarised in Figure 1.

Insert Figure 1 about here

\section{Physical activity promotion practices and determinants of health professionals}


Promotion of PA by health professionals has been shown to significantly improve health, physical and psycho-social outcomes for a variety of patient groups [12, 3]. The promotion of PA by a health professional to their cancer patients has demonstrated significant increases in patients' PA levels, energy expenditure, aerobic fitness, muscular strength and quality of life, as well as reducing the number of barriers to PA [2, 4]. Jones et al. also reported significantly larger increases in PA levels if patients remembered the nature of the PA advice from the health professional [4]. However, relatively little is known about the PA promotion practices of health professionals and the factors that influence this promotion to their cancer patients [5, 6, 18, 21].

In relevant work, studies have reported that up to $40 \%$ of health professionals only promote PA to the patients they feel would benefit from being more physically active, rather than promoting PA as a standard part of usual care for all patients [5, 6, 18, 21]. While these results are somewhat encouraging, the survey response rates were $14-62 \%$ which may indicate a potential response bias, whereby health professionals who regularly promote PA to their patients may have more likely completed the surveys [5, 6, 18, 21]. These findings, therefore should be interpreted with some caution. Nonetheless, the surveys revealed that many health professionals were aware that PA could reduce the physical side-effects of treatment, lower the risk of developing other chronic conditions and improve physical function, mental health and quality of life. Of the health professionals who promoted PA, they also endorsed evidence-based aerobic activities such as walking for weight loss, cardiovascular health, improved mental health and quality of life [16]. Considerably fewer promoted resistance training as a form of PA to their patients, despite resistance training being more beneficial than aerobic activity in counteracting the substantial declines in muscle mass, strength and physical performance seen with common cancer treatments [7]. Most importantly, the survey data revealed that there are many barriers to PA promotion for these health professionals. For example, health professionals thought that other professionals were promoting PA to the patients, reported lacking the time needed for such discussions during patient consultations, and indicated that they lacked the required knowledge in PA promotion [5, 6, 18, 21]. These barriers appeared quite similar to the concerns reported by 236 cancer patients about their regular consultations with their health professionals [1]. Brandes et al. [1] reported that patients indicated their primary barriers to discussing their concerns with health professionals related to the health professionals' behaviour (e.g. a lack of empathy, not inviting the patients' to identify their concerns and an inability to provide accurate information) as well as the consultation environment (e.g. lack of time to listen to concerns). 
Taken together, these findings warrant health professionals being more proactive in the promotion of PA to their patients. This may be achieved by health professionals initiating such discussions with their patients, attentively responding to questions from their patients and assisting their patients develop strategies to overcome any barriers that may make regular PA difficult. However, there is still much to learn about the primary determinants influencing health professionals’ promotion of PA to their patients. If these determinants are better understood, behavioural interventions using evidence-based behavioural change models can assist health professionals’ in promoting healthy behaviours like PA and result in significantly improved outcomes for their patients.

\section{Possible ways to improve the physical activity promotion practices of health professionals}

When discussing PA and other healthy behaviours with their patients, it is recommended that health professionals utilise evidence-based behavioural change practices [3]. In an extensive review of approaches to improve patient-physician communication, Woulda and van de Weil proposed using an adapted version of the reflective-impulsive model of social behaviour [22]. While Woulda and van de Wiel [22] acknowledge that the full implementation of the reflective impulsive model requires considerable effort, they recommended its use as a benchmark for improving patient-physician communication and identified five key learning objectives and teaching methods for communication education. Specific examples of how health professionals may use this approach when discussing PA with their cancer patients are highlighted in Table 1.

Insert Table 1 about here

As alluded to in Table 1, it is understood that the behaviour, knowledge and/or work environment of the health professional may act as facilitators or barriers to effective patient-centred communication about PA. Many health professionals may identify aspects of their own behaviour or work environment that would act as barriers to effective PA communication with their patients, therefore we recommend the development of referral networks, whereby patients are referred to specialists in PA (e.g. exercise physiologists or physiotherapists or psychosocial/ behavioural counselling).

The reflective impulsive model [22] also has major implications for training models used by universities and hospitals. It is recommended that universities and hospitals provide a series of educational experiences which progressively develop the clinical communication skills of their students, interns and staff 
[22, 23]. An overview of how this may be applied to improving PA promotion and counselling for cancer patients is provided in Table 2 .

Insert Table 2 about here

\section{Conclusions}

Health professionals appear interested in promoting PA to their patients but evidence suggests that they experience several barriers to this activity. It is vital that health professionals use evidence-based behavioural change approaches in promoting the proven benefits of PA during their patient consultations. Universities, hospitals and accreditation bodies can all play important roles in assisting health professionals achieve this goal.

More research is required, to continually improve practice in this area. Relevant studies may examine: patient benefits obtained through behavioural theory-based PA promotion programs led by primary cancer clinicians such as oncologists; the determinants and current PA promotion practices of a wider variety of health professionals; and how differences in health professional and patient characteristics may impact PA promotion and patient outcomes.

Based on the strong evidence for the benefits of PA for cancer survivorship [7, 11, 16], health professionals, universities, hospitals and national healthcare accreditation organisations are encouraged to consider the conversational value and implementation processes of PA promotion as part of their usual care for all cancer patients, not just those for those who are subjectively assessed by health professionals to need it. We recommend that evidence-based practice educational modules include discussions on the benefits of PA as well the behavioural change models that may best increase PA levels for cancer patients. Hospitals should examine ways they can better encourage their primary cancer clinicians to work more collaboratively with PA and counselling specialists in this endeavour. This may be achieved by hospitals developing improved PA resources and/or referral pathways to cancer-specific PA programs that are more accessible to patients. Collectively, these approaches may improve PA promotion by health professionals and result in improved outcomes for patients $[14,19]$. 
Conflicts of interest: none.

\section{Figure captions}

Figure 1: Possible role of health professionals in promoting physical activity and improving outcomes for cancer patients. 


\section{References}

1. Brandes, K., A. J. Linn, E. G. Smit, and J. C. M. van Weert. 2015. Patients’ reports of barriers to expressing concerns during cancer consultations. Patient Education and Counseling 98 (3):317-322. doi:10.1016/j.pec.2014.11.021.

2. Damush, T. M., A. Perkins, and K. Miller. 2006. The implementation of an oncologist referred, exercise selfmanagement program for older breast cancer survivors. Psycho-Oncology 15 (10):884-890. doi:10.1002/pon.1020.

3. Gagliardi, A. R., G. Faulkner, D. Ciliska, and A. Hicks. 2015. Factors contributing to the effectiveness of physical activity counselling in primary care: A realist systematic review. Patient Education and Counseling 98 (4):412-419. doi:10.1016/j.pec.2014.11.020.

4. Jones, L. W., K. S. Courneya, A. S. Fairey, and J. R. Mackey. 2004. Effects of an oncologist's recommendation to exercise on self-reported exercise behavior in newly diagnosed breast cancer survivors: a single-blind, randomized controlled trial. Annals of Behavioral Medicine 28 (2):105-113.

5. Jones, L. W., K. S. Courneya, C. Peddle, and J. R. Mackey. 2005. Oncologists' opinions towards recommending exercise to patients with cancer: a Canadian national survey. Supportive Care in Cancer 13 (11):929-937. doi:10.1007/s00520-005-0805-8.

6. Karvinen, K. H., S. McGourty, T. Parent, and P. R. Walker. 2012. Physical activity promotion among oncology nurses. Cancer Nursing 35 (3):E41-E48. doi:10.1097/NCC.0b013e31822d9081.

7. Keogh, J. W. L., and R. D. MacLeod. 2012. Body composition, physical fitness, functional performance, quality of life and fatigue benefits of exercise for prostate cancer patients: a systematic review. Journal of Pain and Symptom Management 43 (1):96-110. doi:10.1016/j.jpainsymman.2011.03.006.

8. Keogh, J. W. L., A. Patel, R. D. MacLeod, and J. Masters. 2013. Perceptions of physically active men with prostate cancer on the role of physical activity in maintaining their quality of life: possible influence of androgen deprivation therapy. Psycho-Oncology 22:2869-2875. doi:10.1002/pon.3363.

9. Keogh, J. W. L., A. Patel, R. D. MacLeod, and J. Masters. 2014. Perceived barriers and facilitators to physical activity in men with prostate cancer: possible influence of androgen deprivation therapy. European Journal of Cancer Care 23 (2):263-273. doi:10.1111/ecc.12141.

10. LeMasters, T. J., S. S. Madhavan, U. Sambamoorthi, and S. Kurian. 2014. Health behaviors among breast, prostate, and colorectal cancer survivors: a US population-based case-control study, with comparisons 
by cancer type and gender. Journal of Cancer Survivorship 8 (3):336-348. doi:10.1007/s11764-0140347-5.

11. Mishra, S. I., R. W. Scherer, P. M. Geigle, D. R. Berlanstein, O. Topaloglu, C. C. Gotay, and C. Snyder. 2012. Exercise interventions on health - related quality of life for cancer survivors. Cochrane Database of Systematic Reviews 8. doi:10.1002/14651858.CD007566.pub2.

12. Orrow, G., A.-L. Kinmonth, S. Sanderson, and S. Sutton. 2012. Effectiveness of physical activity promotion based in primary care: systematic review and meta-analysis of randomised controlled trials. BMJ 344.

13. Ottenbacher, A., R. Sloane, D. C. Snyder, W. Kraus, L. Sprod, and W. Demark-Wahnefried. 2013. Cancerspecific concerns and physical activity among recently diagnosed breast and prostate cancer survivors. Integrative Cancer Therapies 12 (3):206-212. doi:10.1177/1534735412449734.

14. Pinto, B. M., and J. T. Ciccolo. 2011. Physical activity motivation and cancer survivorship. Recent Results in Cancer Research 186:367-387. doi:10.1007/978-3-642-04231-7_16.

15. Robertson, L., R. Richards, R. Egan, and E. A. Szymlek-Gay. 2013. Promotion and support of physical activity among cancer survivors: a service provider perspective. Psycho-Oncology 22 (2):441-446. doi:10.1002/pon.3032.

16. Rock, C. L., C. Doyle, W. Demark-Wahnefried, J. Meyerhardt, K. S. Courneya, A. L. Schwartz, E. V. Bandera et al. . 2012. Nutrition and physical activity guidelines for cancer survivors. CA: A Cancer Journal for Clinicians 62 (4):242-274. doi:10.3322/caac.21142.

17. Schmitz, K. H., R. M. Speck, S. A. Rye, T. DiSipio, and S. C. Hayes. 2012. Prevalence of breast cancer treatment sequelae over 6 years of follow-up. Cancer 118 (S8):2217-2225. doi:10.1002/cncr.27474.

18. Spellman, C., M. Craike, and P. Livingston. 2014. Knowledge, attitudes and practices of clinicians in promoting physical activity to prostate cancer survivors. Health Education Journal 73 (5):566-575. doi:10.1177/0017896913508395.

19. Stacey, F. G., E. L. James, K. Chapman, K. S. Courneya, and D. R. Lubans. 2015. A systematic review and meta-analysis of social cognitive theory-based physical activity and/or nutrition behavior change interventions for cancer survivors. Journal of Cancer Survivorship 9 (2):305-338. doi:10.1007/s11764014-0413-z.

20. Trikalinos, T. A., L. S. Wieland, G. P. Adam, A. Zgodic, and E. E. Ntzani. 2014. AHRQ Comparative Effectiveness Reviews. In Decision Aids for Cancer Screening and Treatment. Rockville (MD): Agency for Healthcare Research and Quality (US). 
21. Williams, K., R. J. Beeken, A. Fisher, and J. Wardle. in press. Health professionals' provision of lifestyle advice in the oncology context in the United Kingdom. European Journal of Cancer Care. doi:10.1111/ecc.12305.

22. Wouda, J. C., and H. B. M. van de Wiel. 2013. Education in patient-physician communication: How to improve effectiveness? Patient Education and Counseling 90 (1):46-53. doi:10.1016/j.pec.2012.09.005.

23. Wouda, J. C., and H. B. M. van de Wiel. 2014. The effects of self-assessment and supervisor feedback on residents’ patient-education competency using videoed outpatient consultations. Patient Education and Counseling 97 (1):59-66. doi:10.1016/j.pec.2014.05.023. 
Fig. 1

Cancer patients live with many sideeffects from treatments and disease

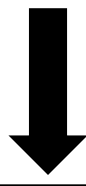

Physical activity can alleviate many treatment and disease related side-effects

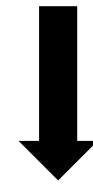

Many cancer patients are not meeting recommended physical activity guidelines

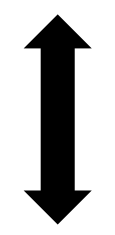

Cancer organisations agree cancer patient should engage in physical activity
More health professionals are becoming aware of the general benefits of physical activity for their cancer patients and wish to promote these benefits
Health professionals may be able to assist cancer patients increase their physical activity levels

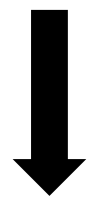

However, many health professionals may have limited knowledge of physical activity counselling and many barriers to its promotion 
Table 1.

Learning objectives for improving health professional-patient communication regarding physical activity: A proposed approach based on the reflective impulsive model of social behaviour [19].

Learning objectives

Goal directed communication

Control over the conversation and relationship

Eliciting and synthesising information

Conveying information

Developing and implementing a shared plan of care
Examples of how health professionals may implement this approach

Ensure that the promotion of PA and the discussion of potential barriers, facilitators, risk and benefits are considered a priority for discussion on a regular basis.

Develop a consensus with the patient on the primary and secondary goals of each consultation, how discussions of PA may be relevant to these goals and respond flexibly to any concerns raised by the patient.

Be attentive when listening to the patient so to get an overview of the patient's concerns as well as their perceptions on the determinants of these concerns and the impact these have on their cancer survivorship. Determine what issues raised by the patient may be improved by PA and/or what strategies could be used to increase PA levels in these patients if they can identify barriers to their PA engagement.

Provide an overview of the evidence based literature of the benefits of PA for cancer survivorship in a simple and straightforward manner. As this level of PA understanding may be beyond some health professionals, it is recommended that hospitals and healthcare systems look to develop better referral networks between cancer clinicians and PA specialists such as exercise physiologists and physiotherapists who may provide more detailed PA prescription details and/or offer cancer specific PA or rehabilitation classes.

Discussion of PA should be regularly scheduled with at least one member of the health professional healthcare team to assist the patient regularly perform PA. This should involve the primary cancer clinician and if required the PA and psycho-oncology specialists. Regardless of whose primary responsibility this is, a shared plan needs to be developed between the patient and relevant health professionals in regards to the primary and secondary goals of the PA program as well as developing strategies to overcome some of the barriers to regular PA participation. 
Table 2.

Teaching and assessment methods of clinical communication skills with respect to patient physical activity: A proposed approach for universities and training hospitals based on the reflective impulsive model of social behaviour [19, 20].

\begin{tabular}{|c|c|c|}
\hline Teaching methods & Setting & Examples of how universities and/or hospitals may implement this approach \\
\hline Assignments & Universities & $\begin{array}{l}\text { Utilise assignments whereby health professional students are provided case studies and are required to practice } \\
\text { short conversations with peers, relatives or patients on issues whereby PA may be a relevant adjunct therapy. } \\
\text { Audio or video taping of these communication scenarios would be useful to collect as it will allow the students } \\
\text { to obtain objective feedback on their performance and allow them to reflect on ways in which to improve in } \\
\text { the future. }\end{array}$ \\
\hline Simulation & Universities & $\begin{array}{l}\text { This can involve role-play, feedback and small groups involving communication scenarios whereby PA may } \\
\text { be a relevant adjunct therapy. Students should receive frequent opportunities for feedback, reflection and } \\
\text { correction as they are important components of this process. }\end{array}$ \\
\hline Workplace based learning & Hospitals & $\begin{array}{l}\text { The use of video-on-the-job whereby the health professional can view videotapes of their outpatient and } \\
\text { clinical consultations and perhaps feedback from supervisory staff is a crucial aspect of workplace based } \\
\text { learning [20]. The focus when viewing these video recordings could be on the general communication style, } \\
\text { the frequency with which PA was discussed where relevant, and the accuracy of the PA information provided } \\
\text { as well as the use of relevant behavioural change theories in promoting PA participation. }\end{array}$ \\
\hline
\end{tabular}

